\title{
Cloning and Characterization of Two MAPK Genes UeKpp2 and UeKpp6 in Ustilago esculenta
}

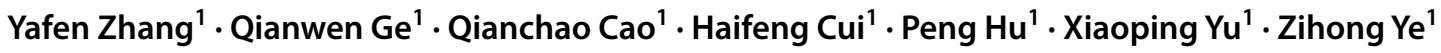

Received: 24 October 2017 / Accepted: 24 March 2018 / Published online: 28 March 2018

(c) The Author(s) 2018

\begin{abstract}
Ustilago esculenta, resembling a fungal endophyte in Zizania latifolia, inhibits the host plant flowering and induces the host stems to swell and form edible galls. It is well believed that when and how the fungus infects and proliferates in the host plants during the host development is of importance in the edible gall formation. Mitogen-activated protein kinases (MAPKs) have been found to play an important role in sensing environment cues and regulating infection. Two MAPK genes UeKpp2 and UeKpp6 from U. esculenta were cloned and suggested to be involved in the Fus3/Kss1 pathway by a phylogenetic analysis with the neighbor-joining method. Quantitative RT-PCR (qRT-PCR) analyses indicated that expression of UeKpp2 and UeKpp6 were induced during mating and infection processes, and their expression patterns displayed differentially under different carbon and nitrogen sources. In addition, subcellular localization of UeKpp2 or UeKpp6 fused with the reporter green fluoresce protein was observed by confocal laser scanning microscope, and yeast two-hybrid assays were carried out. Results showed that both UeKpp2 and UeKpp6 were located in cytoplasm and interacted with UePrf1, indicating their involvement in hyphal growth and host-pathogen regulation. Only UeKpp2 but not UeKpp6 interacted with the upstream MAPK kinase UeFuz7, implying an additional MAPK pathway, in which UeKpp6 involved, existed.
\end{abstract}

\section{Introduction}

Ustilago esculenta, belonging to the Ustilaginaceae of basidiomycetes, resembles an endophytic fungus of Zizania latifolia, a perennial aquatic grass widely cultivated in southeastern Asian countries [1]. The U. esculenta-infected host plants, commonly called jiaobai in China, fail to flower and produce seeds, whereas the plant upper stems swell and form edible galls. In the fields, the majority of swollen galls with white appearance and soft tissues, named white jiaobai, are consumed as an aquatic vegetable for its flavor and delicacy [2]. But galls filled with dark-colored teliospores, called grey jiaobai, always appear in a harvest season [3].

Yafen Zhang and Qianwen Ge contributed equally to this work.

Electronic supplementary material The online version of this article (https://doi.org/10.1007/s00284-018-1483-3) contains supplementary material, which is available to authorized users.

Zihong Ye

zhye@cjlu.edu.cn

Zhejiang Provincial Key Laboratory of Biometrology and Inspection \& Quarantine, College of Life Sciences, China Jiliang University, Hangzhou 310018, Zhejiang, China
They are often discarded because they seriously distort the quality of jiaobai. Previous studies reported that the strains of $U$. esculenta from white jiaobai and grey jiaobai were distinct, characterized as mycelia-teliospore (MT) and teliospore (T) strains [4], respectively. It was speculated that the strains of MT and T are different in pathogenicity or teliospore formation [4].

It is generally accepted that mating of two compatible haploid strains to form invasive hyphae is essential for fungal infection in Ustilaginaceae [5]. During mating and hyphal formation, MAPK signaling cascades (consist of MAP kinase kinase kinase (MAPKKK), MAP kinase kinase (MAPKK), and MAP kinase (MAPK) sequentially) play crucial roles in the transduction of extracellular cues and fungal pheromone response [6, 7]. Besides, MAPKs show different functions. For instance, in Saccharomyces cerevisiae, Fus3 regulates inter gametophytic mating and Kss 1 is primarily responsible for the following invasion [8]. In Candida albicans, Cek1 and Cek2 have overlapping functions in the mating progress and filamentous growth [9]. In Ustilago maydis, which is closely related to U. esculenta, Ubc3/Kpp2, and Kpp6 has been proven to function in mating and plant infection [10, 11]. Fuz7, which functions upstream of Kpp2 and Kpp6 [12], plays an important role in conjugation tube 
and filament formation, and the maintenance of filamentous growth in $U$. maydis [13]. The pheromone response factor Prf1, which could be phosphorylated by Kpp2 and Kpp6 [14], is critical in mating, filamentous growth, and pathogenicity in $U$. maydis [15]. In addition, Ubc $3 / \mathrm{Kpp} 2$ could be phosphorylated by activated Fuz7 and then regulate both the prf1-dependent transcriptional response to pheromone and the prf1-independent formation of conjugation tube [12]. Furthermore, the distinct N-terminus of Kpp6 has been proven having an inhibitory function in pathogenicity, in which a PR-motif (proline-rich motif) is located to interact with the SH3 domain of Sho1 [16]. What MAPKK activates Kpp6, however, remains unknown [17, 18].

Recently, the differentiations of MT and T strains were deeply investigated. Growth and pathogenicity defects in MT strains were discovered and a distinct mating response to different culture media and culture time between the MT and $T$ type strains were proved [19]. In this report, in order to discover the different mating response mechanisms that determine pathogenicity difference, we cloned two MAP kinase genes UeKpp2 and UeKpp6 from U. esculenta, and analyzed their relative expression during mating/infection progresses and under different exogenous stimuli. In addition, we employed a yeast two-hybrid assay to study the interaction between UeKpp2/UeKpp6 and UeFuz7/UePrf1. We also fused UeKpp2/UeKpp6 to the reporter gene EGFP to investigate the subcellular localization of UeKpp2 and UeKpp6 during the mating process.

\section{Materials and Methods}

\section{Strains and Growth Conditions}

Compatible haploid $U$. esculenta strains UeMT10 (a3b3 CCTCC AF 2015020)/UeMT46 (a2b2 CCTCC AF 2015021) isolated from white jiaobai and UeT14 (a1b1 CCTCC AF 2015016)/UeT55 (a2b2 CCTCC AF 2015015) isolated from grey jiaobai were grown at $28{ }^{\circ} \mathrm{C}$ on the YEPS (1\% yeast extract, $2 \%$ peptone, and $2 \%$ sucrose) solid medium. The S. cerevisiae strain Y2HGold and Y187 (Clontech) were used for two-hybrid interaction studies. The tubular stems of Z. latifolia (longjiao 2\#) plants were dug up from field and planted into a mixed soil (nutritive soil: vermiculite: perlite 4:4:1) in greenhouse under a $8 \mathrm{~h}$ light $\left(\sim 800 \mu \mathrm{mol} \mathrm{s}{ }^{-1} \mathrm{~m}^{-2}\right.$ photons $\mathrm{m}^{-2} \mathrm{~s}^{-1}$ of intensity) and $16 \mathrm{~h}$ dark cycle at $15 \pm 2{ }^{\circ} \mathrm{C}$ with $80 \%$ relative humidity until the tubular stems grow to tillering stage. Then change the conditions to a $12 \mathrm{~h} \mathrm{light}$ and $12 \mathrm{~h}$ dark cycle at $25 \pm 2{ }^{\circ} \mathrm{C}$ with $80 \%$ relative humidity. Stem tip samples were obtained from the three important periods, tillering stage (the day before the culture conditions change, $\sim 20$ days after tubular stems germination), 8-leaf stage ( $\sim 20$ days after the culture conditions changed), swollen stage ( 40 days after the culture conditions changed), respectively.

\section{Gene Cloning}

The genomic DNA was extracted with Ezup Column Fungi Genomic DNA Purification Kit (Sangon Biotech, China). Total RNA was isolated according to the RNAiso Plus' instructions (Takara, Japan). The cDNAs were synthesized using PrimeScript ${ }^{\mathrm{TM}}$ II 1 st strand cDNA Synthesis Kit (Takara, Japan). Specific primers (supplementary data 1) were designed according to genome sequencing data. PCR was performed with LA Taq DNA polymerase (Takara, Japan). The objective PCR products were purified with SanPrep Column DNA Gel Extraction Kit (Sangon Biotech, China) and cloned into pMD19-T (Takara, Japan), and then confirmed by sequencing.

\section{Bioinformatics Analysis}

The open reading frame (ORF) of UeKpp2 and UeKpp6 were determined using the ORF finder (http://www.ncbi. nlm.nih.gov/gorf/). The conserved motifs and predicted domains were analyzed at NCBI. Multiple alignments of the putative proteins were performed with the DNAMAN 8.0 software. Phylogenetic analysis of MAP kinases from other related fungi was performed with the MEGA 5.0 program using the neighbor-joining method.

\section{Mating Assays}

Haploid strains UeMT10, UeMT46 and UeT14, UeT55 were cultured in the YEPS liquid medium to an $\mathrm{OD}_{600}$ of 1.0-1.5, and then collected and resuspended in the YEPS liquid medium to an $\mathrm{OD}_{600}$ of 2.0. After compatible haploid strains mixed, $3 \mu \mathrm{l}$ of cells was dropped to culture on the YEPS solid medium or a prepared medium with indicated compounds (with different carbon or nitrogen sources described below) at $28{ }^{\circ} \mathrm{C}$. Samples were collected at $0,12,24,36$, 48, $60 \mathrm{~h}$. After $60 \mathrm{~h}$, mating colonies were observed with microscopy. Media for treatments were prepared with basic medium (BM) and indicated carbon or nitrogen sources. Basic medium was prepared as follows: $\mathrm{K}_{2} \mathrm{HPO}_{4} 1 \mathrm{~g}$, $\mathrm{MgSO}_{4} \cdot 7 \mathrm{H}_{2} \mathrm{O} 0.5 \mathrm{~g}, \mathrm{FeSO}_{4} \cdot 7 \mathrm{H}_{2} \mathrm{O} 0.01 \mathrm{~g}, \mathrm{KCl} 0.5 \mathrm{~g}$, Agar $15 \mathrm{~g}$, adding distillated water to $1000 \mathrm{ml}$, and autoclaving for sterilization. Nitrogen source $\left(\mathrm{NH}_{4} \mathrm{NO}_{3}\right.$, methionine, arginine, or urea) was added to $\mathrm{BM}$ to make the $\mathrm{c}(\mathrm{N})=20 \mathrm{mM} / \mathrm{l}$, filtered by Millipore filters $(0.22 \mu \mathrm{m})$, with sucrose as carbon source $(50 \mathrm{mM} / \mathrm{l})$, being nitrogen sources changed medium. Carbon source (galactose, glucose, fructose, sorbitol, mannitol, lactose, or maltose) was added to BM to make the c $(C)=600 \mathrm{mM} / \mathrm{l}$, filtered by Millipore filters $(0.22 \mu \mathrm{m})$, with $\mathrm{KNO}_{3}$ as nitrogen source $(20 \mathrm{mM} / \mathrm{l})$, being carbon 
sources changed medium. The $\mathrm{BM}$ with $20 \mathrm{mM} / 1 \mathrm{KNO}_{3}$ and $50 \mathrm{mM} / 1$ sucrose was prepared as blank control (CK).

\section{Expression Analysis by qRT-PCR}

The cDNAs were synthesized using the PrimeScript ${ }^{\mathrm{TM}}$ RT reagent Kit with gDNA Eraser (Takara, Japan). qPT-PCR was performed with SYBR Premix EX Taq ${ }^{\mathrm{TM}}$ (TliRNaseH Plus) (Takara, Japan) on the ABI 7500 Real-time Detection System (Applied Biosystems, USA) using the gene-specific RT primer pairs (supplementary data 1 ). $\beta$-Actin was used as the internal reference for measuring gene expression. The comparative CT $\left(2^{-\Delta \Delta \mathrm{CT}}\right)$ method was used for calculating relative gene expression [20].

\section{Subcellular Localization of MAPK-GFP Chimeric Proteins}

The MAPK-eGFP chimeric fragments were gained by fusion PCR with specific primers (Supplementary data 1) and linked by the PG linker proteins [21]. Then recombinant plasmid pUMa932-UeKpp2/UeKpp6-eGFP was constructed and cut to linear segments by QuickCut ${ }^{\mathrm{TM}}$ NdeI (Takara, Japan) for next transformation into UeT14 haploid strain using an efficient genetic manipulation protocol reported before [22]. GFP fluorescence was observed using a confocal laser scanning microscope (Leica Microsystems, USA).

\section{Yeast Two-Hybrid Assay}

Yeast two-hybrid assay was performed by the Matchmaker ${ }^{\mathrm{TM}}$ Gold Yeast Two-Hybrid System (Clontech). The coding regions of UeKpp2 and UeKpp6 were cloned into the bait vector pGBKT7 and transformed into yeast strain Y2HGold. The coding regions of $\mathrm{UeFuz} 7$ and $\mathrm{UePrfl}$ were cloned into the prey vector $\mathrm{pGADT} 7$ and transformed into yeast strain Y187. Subsequently Y2HGold and Y187 carrying recombinant plasmids were mixed and plated on the SD-Leu-Trp medium for initial selection. Transformants were selected randomly and streaked on the SD/-Leu/-Trp/-His/-Ade/X- $\alpha$ Gal medium for a protein-protein interaction test.

\section{Statistical Analysis}

All experiments were performed in triplicates and data were shown as mean \pm SD from the three independent experiments. All data were analyzed according to the Duncan's method. The probability values of $P<0.05$ were considered as significant.

\section{Results}

\section{Identification of Two MAP Kinases UeKpp2 and UeKpp 6 in U. esculenta}

BlastP searches against the genomic database of $U$. esculenta (JTLW00000000) were performed using the Kpp2 and Kpp6 protein sequences of $U$. maydis as queries. Two predicted loci (g262 with 96\% identity to Kpp2, and g4166 with 83\% identity to Kpp6) were obtained. The full-length cDNAs of the two predicted genes were cloned and confirmed by sequencing. One encoding a protein of 354 amino acids (Supplementary data 2A) with a high identity to Kpp2, was named UeKpp2 (Accession Number: KU855052). UeKpp2 has an estimated molecular mass of $90.56 \mathrm{kDa}$ and a predicted $\mathrm{pI}$ of 5.00. Another gene coding for 578 amino acids (Supplementary data 2B) with a high identity to Kpp6 was named UeKpp6 (accession number: KU855053). UeKpp6 possesses an estimated molecular mass of $148.52 \mathrm{kDa}$ with a predicted $\mathrm{pI}$ of 4.88 . There is no intron in UeKpp 2 and UeKpp6, and their encoding amino acid sequences were identical in the MT and T type strains.

Furthermore, the STKc_ERK1_2_like family region and conserved TEY dual phosphorylation sites, which have been shown to be phosphorylated upon stimulation through its MAPK kinases [23], were found in UeKpp2 and UeKpp6 (Supplementary data 2). Interestingly, being similar to what was found in $U$. maydis [11], an additional $\mathrm{N}$-terminal domain with a perfect match box to the Prf1 consensus binding site (ACAAAGGGA) exists in both proteins. An alanine linker encoded by a trinucleotide repeat domain (GCT)n found in Kpp6 [11], does not exist in UeKpp6.

Sequence alignment and phylogenetic tree analysis revealed that UeKpp2 showed more than $96 \%$ of identity to Kpp2 from smut fungi [10, 24], 55-75\% of identity to other homologs in pathogenic fungi, such as Pmk1 in M. grisea [25], Fus3p [26], and Kss1p [8] in S. cerevisiae, Cek1 in C. albicans [27], whereas UeKpp6 showed a lower identity, nearly $80 \%$ to Kpp6 from smut fungi, 35\% to other MAP kinases. The two MAP kinases were both clustered into Fus3/Kss1 pathway (Fig. 1), and showed high levels of similarity to smut fungi such as $U$. maydis, Ustilago hordei, Sporisorium reilianum (Fig. 1 and Supplementary data 3), implying that they might be involved in mating response and pathogenicity [7].

\section{The Expression of UeKpp2 and UeKpp6 was Induced During Mating and Infection Progresses}

To explore the possible roles of UeKpp2 and UeKpp6 in mating response and pathogenicity, expression patterns of 


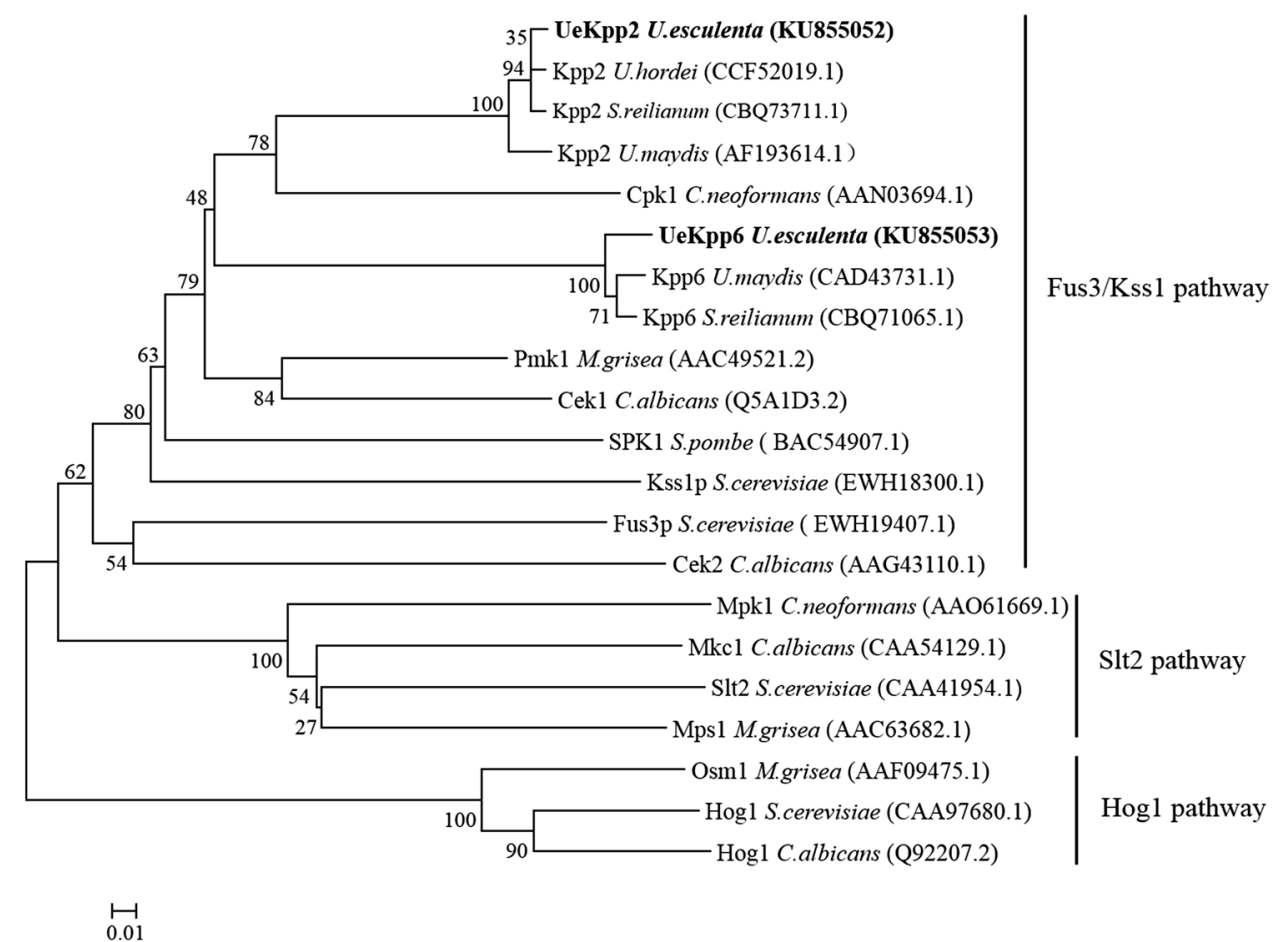

Fig. 1 Phylogenetic tree constructed from the MAP kinase protein sequences of several fungi. Amino acid sequences were used and analyzed by MEGA 5.0 with the neighbor-joining method. Numbers on the branches represent bootstrap support for 1000 replicates. The related MAPK proteins used were: Ustilago esculenta UeKpp2(KU855052) and UeKpp6 (KU855053), marked in bold; Ustilago hordei Kpp2 (CCF52019.1); Sporisorium reilianum Kpp2 (CBQ73711.1) and Kpp6 (CBQ71065.1); Ustilago may-

UeKpp 2 and UeKpp6 during mating and infection progresses were analyzed. In the $\mathrm{T}$ type strains, hyphae were observed at $12 \mathrm{~h}$ and the majority of white and fuzzy filaments appeared at $36 \mathrm{~h}$, while the MT type strains showed $12 \mathrm{~h}$ later in hyphae formation and weaker hyphal growth (Fig. 2a). Meanwhile, temporal expression results showed that during mating process, UeKpp2 and UeKpp6 were up-regulated in hyphal growth phase than in cells budding phase, especially after white and fuzzy filaments formation, and showed a distinctly higher expression in the $T$ type strains after the hyphal formation when compared to the MT strains, indicating their involvement in hyphal growth. In addition, relative expression of UeKpp 2 was increasing and peaked at $36 \mathrm{~h}$ in the T type strains but $60 \mathrm{~h}$ in the MT type strains (Fig. 2b), while UeKpp6 increased to the highest expression level at $48 \mathrm{~h}$ (Fig. 2c), indicating an unknown and distinct role of UeKpp2 and UeKpp6 in the MT and T type strains.

To analyze potential roles of UeKpp 2 and UeKpp6 in the fungal infection progress during host growth and development, three related periods of the host plant were selected and the relative expression patterns of UeKpp 2 and UeKpp6 dis Kpp2 (AF193614.1) and Kpp6 (CAD43731.1); Cryptococcus neoformans Cpk1 (AAN03694.1) and Mpk1 (AAO61669.1); Magnaporthe grisea Pmk1 (AAC49521.2), Mps1 (AAC63682.1) and Osm1 (AAF09475.1); Candida albicans Cek1 (Q5A1D3.2), Cek2 (AAG43110.1), Mkc1 (CAA54129.1) and Hog1 (Q92207.2); Schizosaccharomyces pombe SPK1 (BAC54907.1); Saccharomyces cerevisiae Kss1p (EWH18300.1), Fus3p (EWH19407.1), Slt2 (CAA41954.1) and Hog1 (CAA97680.1)

in these three periods were determined. The first period is the tillering stage when the fungi are trying to go into the different seedlings. The second is the 8-leaf stage that is supposed to the key period for the fungi infection and spread. The third is the swollen stage when the fungi are widely distributed throughout the swollen tissues. A remarkable increase of UeKpp6 in the second and key period (Fig. 2e) and high expression of UeKpp2 in the swollen stage were observed (Fig. 2d), indicating their involvement in the interaction between $U$. esculenta and Z. latifolia, especially for UeKpp6.

\section{UeKpp2 and UeKpp6 Showed Different Expression Pattern in Response to Distinct Nitrogen or Carbon Source Treatment}

To find out whether UeKpp2 and UeKpp6 in U. esculenta respond to external stimuli or not, especially during starvation of nutrition [7], distinct nitrogen and carbon source treatment assays were carried out. The results showed that methionine, arginine obviously promoted the hyphal growth of the MT type strains, while $\mathrm{NH}_{4} \mathrm{NO}_{3}$ and arginine inhibited 

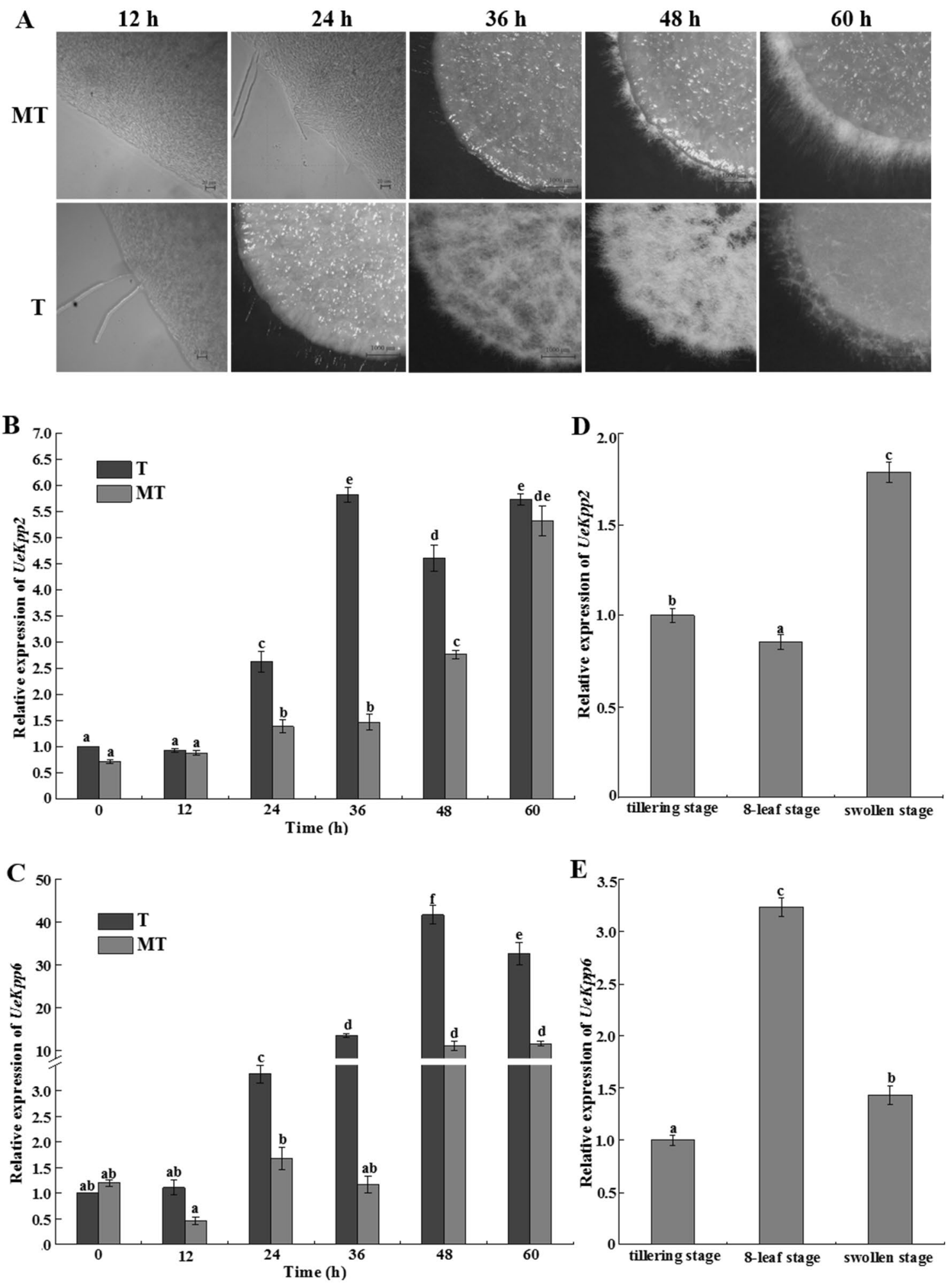

Fig. 2 The relative expression of UeKpp2 and UeKpp6 during mating and infection progress. a Mating phenotype of MT and Type strains at different time with $12 \mathrm{~h}$ intervals. Earlier photos were taken by an ordinary microscope with the bars of $20 \mu \mathrm{m}$; Other images were taken by a stereomicroscope with the bars of $1000 \mu \mathrm{m}$; Relative expression of UeKpp2 (b) and UeKpp6 (c) were analyzed dur- ing different mating time; Relative expression of UeKpp2 (d) and UeKpp6 (e) during infection progress were also analyzed. $\beta$-Actin was used as an internal control. Data were reported as means \pm SD of three repeated samples. All data were subjected to statistical analyses according to the Duncan's method. The probability values of $P<0.05$ were considered as significant and reported with the letter notation 

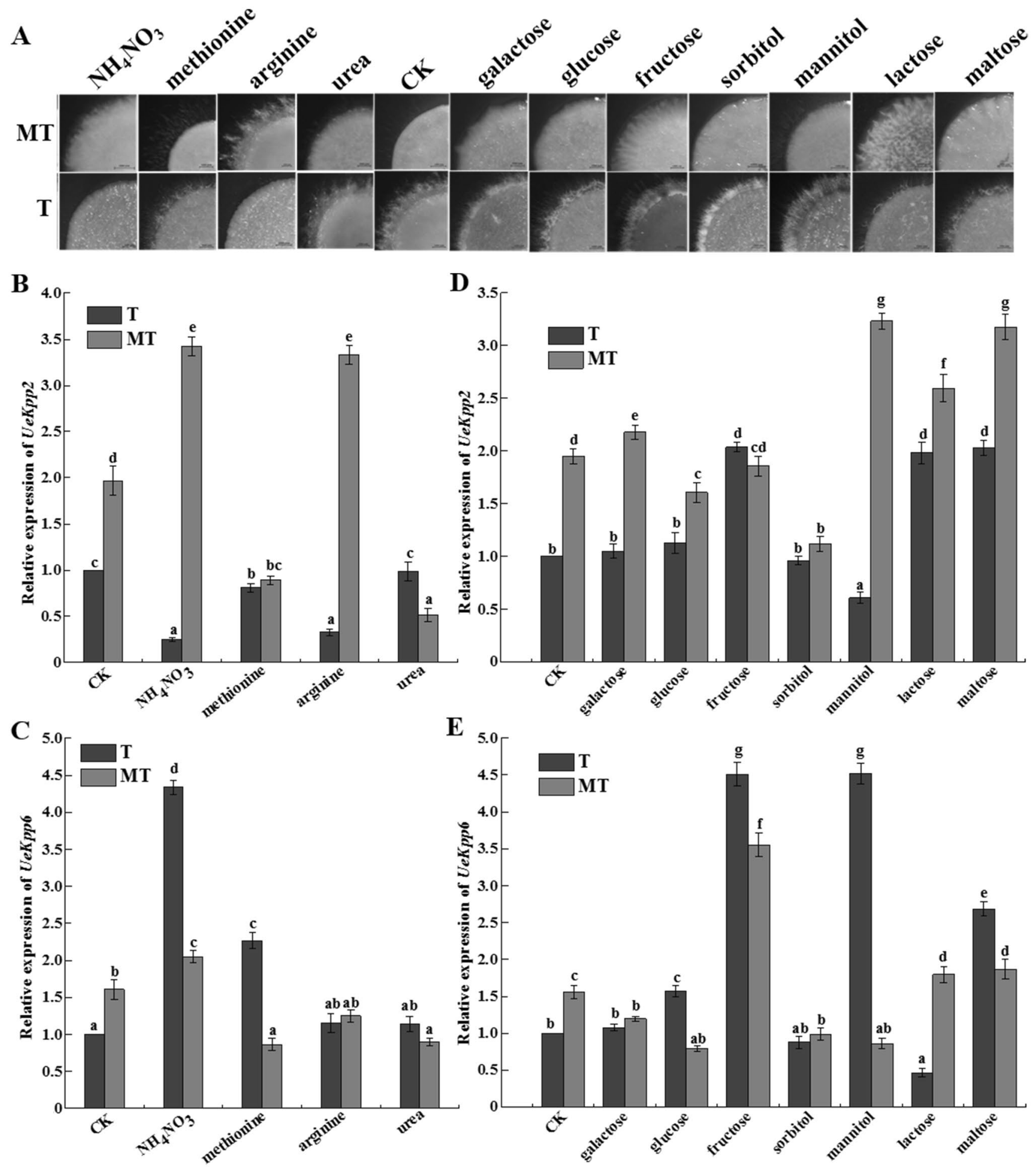

Fig. 3 The expression patterns of UeKpp2 and UeKpp6 under distinct nitrogen or carbon source treatments. a Mating phenotype of MT and $\mathrm{T}$ type strains on a solid culture medium with different nitrogen and carbon sources. Images were taken after 3 days by a stereomicroscope. Bars indicate $1000 \mu \mathrm{m}$. Relative expression of UeKpp2 (b) and UeKpp6 (c) in distinct nitrogen sources were analyzed; Also, Relative expression of UeKpp2 (d) and UeKpp6 (e) were analyzed in dis-

tinct carbon source treatment. The relative expression in the control medium with sucrose $(50 \mathrm{mM})$ and $\mathrm{KNO}_{3}(20 \mathrm{mM})$ was taken as one fold relative expression sample. $\beta$-Actin was used as an internal control. Data were reported as means \pm SD of three repeated samples. All data were subjected to statistical analyses according to the Duncan's method. The probability values of $P<0.05$ were considered as significant and reported with the letter notation 
the hyphal growth of the T type strain (Fig. 3a). The relative expression of UeKpp2 was up-regulated in the MT strains and down-regulated in the T strains significantly, under the stimulus of $\mathrm{NH}_{4} \mathrm{NO}_{3}$ and arginine (Fig. 3b), indicating a consistent association of UeKpp 2 with hyphal growth. However, it showed no difference of expression of UeKpp6 in the $\mathrm{T}$ and MT type strains under various nitrogen source treatments except the stimulus of $\mathrm{NH}_{4} \mathrm{NO}_{3}$ which gave more than threefolds higher expression of UeKpp6 in the T type strains (Fig. 3c).

In addition, the MT strains showed a defect in mating under a variety of carbon sources except mannitol while the $\mathrm{T}$ strains displayed a normal growth (Fig. 3a). As a whole, the expression of UeKpp2 in the MT type strains was more obviously higher than that in the $\mathrm{T}$ type (Fig. 3d). When compared to the control carbon source, the relative expression of UeKpp2 in the MT strains was up-regulated under mannitol, lactose, and maltose treatment but down-regulated under sorbitol treatment, while fructose and disaccharide promoted expression of UeKpp 2 in the T strains (Fig. 3d). Also, fructose, mannitol, and maltose could promote expression of UeKpp6 significantly, while lactose could suppress expression of UeKpp6 in the $\mathrm{T}$ strains (Fig. 3e).

\section{UeKpp2 and UeKpp6 Were Localized in Cytoplasm and Interacted with UePrf1 in Vitro}

In order to reveal the functional mechanism of UeKpp2 and UeKpp6 in U. esculenta, over-expression of pUMa932UeKpp2/UeKpp6-GFP were conducted in UeT14 haploid strain to analyze subcellular localization of UeKpp2 and UeKpp6 under mating conditions. As shown by fluorescence microscopy, both UeKpp2 and UeKpp6 were localized in the cytoplasm, but UeKpp2 showed an aggregated distribution pattern (Fig. 4a, b). In addition, the yeast two-hybrid assays were carried out and results showed that UeKpp2 and UeKpp6 were able to interact with UePrf1 (ALS87609.1) (Fig. 4c), which is in agreement to the function of MAP kinases in U. maydis [12]. However, only UeKpp2 interacted with UeFuz7 (KX369240) (Fig. 4c).
$\mathbf{A}$

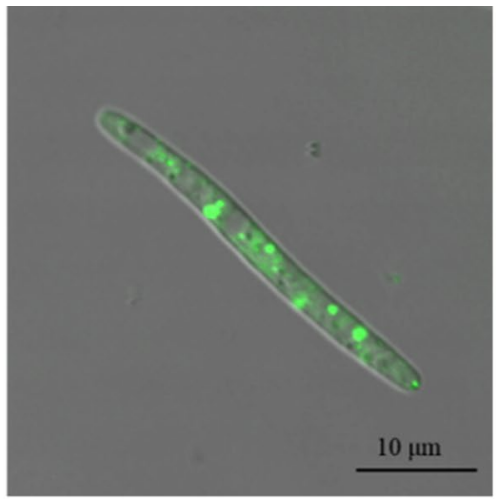

UeT14 UeKpp2

B

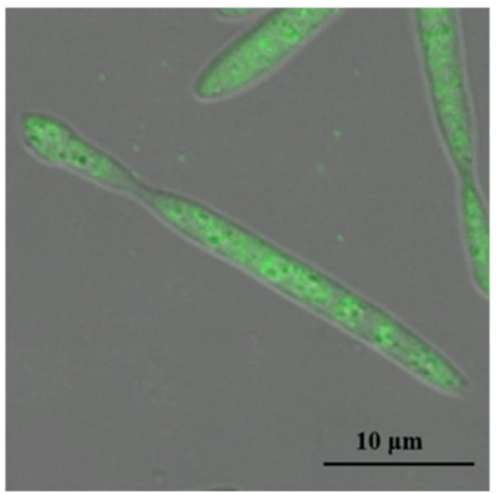

UeT14 UeKpp6

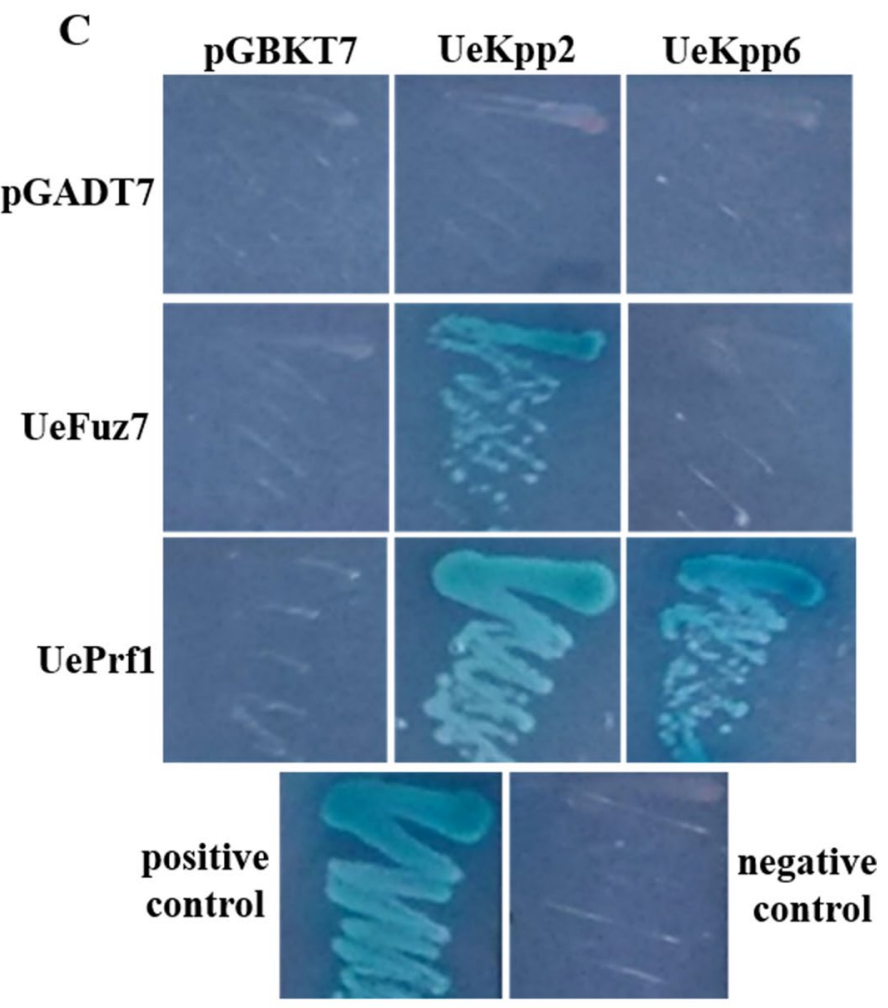

SD/-Leu/-Trp/-His/-Ade/X-a-Gal
Fig. 4 Subcellular location and protein interaction of UeKpp2 and UeKpp6. Subcellular location of UeKpp2 (a) and UeKpp6 (b) were studied in $U$. esculenta. Images were taken by a confocal laser scanning microscope. Bars indicate $10 \mu \mathrm{m}$. $\mathbf{c}$ The interaction between the two MAP kinases and UeFuz7/UePrf1 from $U$. esculenta.
pGBK7 or its derivatives (as indicated on top) were transformated into Y2HGold. pGADT7 and its derivatives (as indicated on left) were transformated into Y187. Y2HGold [pGBKT7-53] and Y187 [pGADT7-T] were used as a positive control. Y2HGold [pGBKT7Lam] and Y187 [pGADT7-T] were used as a negative control 


\section{Discussion}

MAPK signaling pathways play essential roles in many aspects of fungal development, such as mating, appressorium formation, and pathogenesis or virulence [7], especially the Fuz3/Kss 1 pathway, a common pathway in many fungi, such as, U. maydis, M. grisea, and C. albicans. In this study, two MAPK genes UeKpp2 and UeKpp6 from $U$. esculenta were cloned and their putative functions were proposed in the Fuz3/Kss1 pathway because they possess a TEY motif in the activation loop of subdomain VIII (Fig. 1) which is a common character for the Fus3/Kss 1 family [23] (Supplementary data 3). The UeKpp2 expression profile matched well with the hyphal growth in the $\mathrm{T}$ and MT type strains (Fig. 2a, b) and was up-regulated at the later stage during infection (Fig. 2d), while UeKpp6 showed a highly up-regulated expression at the later phase of hyphal growth in T and MT type strains (Fig. 2c) and at an earlier stage in the infection progress (Fig. 2e). The result indicated that, similar to Kpp2 and Kpp6 in U. maydis [10-12], UeKpp 2 might be more related to transmitting pheromone signals, while UeKpp6 might play a more important role in the interaction between $U$. esculenta and Z. latifolia.

In response to the environment cues such as distinct nitrogen and carbon sources, UeKpp2 and UeKpp6 showed more sensitive to nitrogen sources than carbon sources and their responsive patterns were different (Fig. 3). In more details, the expression pattern of UeKpp2 to the arginine and $\mathrm{NH}_{4} \mathrm{NO}_{3}$ treatments indicated its role in mating in vitro, because of its up-regulation in the MT type strains with promoted hyphal growth and down-regulation in the $\mathrm{T}$ type strains with inhibited hyphal growth (Fig. 3a, b). But UeKpp6 might have an undiscovered role in the nitrogen response because in the methionine and $\mathrm{NH}_{4} \mathrm{NO}_{3}$ treatments, the expression of UeKpp6 was up-regulated in the $\mathrm{T}$ type strains with inhibited hyphal growth but down-regulated in the MT type strains with promoted hyphal growth (Fig. 3a, c), which is opposite to the predicted mating regulation roles. We have also found that both UeKpp2 and UeKpp6 were located in cytoplasm and interacted with UePrf1 (Fig. 4), which is similar to the case in $U$. maydis and have been proved to be important in hyphal growth and host-pathogen regulation [12], providing further evidence that UeKpp2 and UeKpp6 participate in hyphal growth and stem swollen induction. The story that only UeKpp2 but not UeKpp6 could interact with UeFuz7 (Fig. 4c), a homolog to Fuz7 in U. maydis [12], indicates that UeKpp6 may be involved in other MAPK cascades in response to environment cues. The detailed function of UeKpp6 in other MAPK cascades needs to be investigated. Therefore, the studies of $U$. esculenta are of importance, particularly in the sense of helping discover distinct MAPK cascades in the interaction system of $U$. esculenta and Z. latifolia, since Z. latifolia was the only host to date in forming edible vegetable because of $U$. esculenta infection [2].

Acknowledgements We thank Prof. Michael Feildbrügge (HeinrichHeine-Universität Düsseldorf, Germany) for sharing the experimental materials and experimental techniques. We thank Prof. Chuanxin Sun (Swedish University of Agricultural Sciences, Sweden) for helping to modify and polish the language. This work was supported by the Natural Science Foundation of Zhejiang Province [LQ15C140003], National Natural Science Foundation of China [31600634 and 31470785].

\section{Compliance with Ethical Standards}

Conflict of interest The authors declare that they have no competing interests.

Open Access This article is distributed under the terms of the Creative Commons Attribution 4.0 International License (http://creativeco mmons.org/licenses/by/4.0/), which permits unrestricted use, distribution, and reproduction in any medium, provided you give appropriate credit to the original author(s) and the source, provide a link to the Creative Commons license, and indicate if changes were made.

\section{References}

1. Guo HB, Li SM, Peng J, Ke WD (2007) Zizania latifolia Turcz. cultivated in China. Genet Resour Crop Evol 54(6):1211-1217. https://doi.org/10.1007/s10722-006-9102-8

2. Zhang JZ, Chu FQ, Guo DP, Hyde KD, Xie GL (2012) Cytology and ultrastructure of interactions between Ustilago esculenta and Zizania latifolia. Mycol Prog 11(2):499-508. https://doi. org/10.1007/s11557-011-0765-y

3. Zhang JZ, Chu FQ, Guo DP, Ojaghian MR, Hyde KD (2014) The vacuoles containing multivesicular bodies: a new observation in interaction between Ustilago esculenta and Zizania latifolia. Eur J Plant Pathol 138(1):79-91. https://doi.org/10.1007/s1065 8-013-0303-7

4. You W, Liu Q, Zou K, Yu X, Cui H, Ye Z (2011) Morphological and molecular differences in two strains of Ustilago esculenta. Curr Microbiol 62(1):44-54. https://doi.org/10.1007/s0028 4-010-9673-7

5. Brefort T, Doehlemann G, Mendoza-Mendoza A, Reissmann S, Djamei A, Kahmann R (2009) Ustilago maydis as a Pathogen. Annu Rev Phytopathol 47(1):423-445. https://doi.org/10.1146/ annurev-phyto-080508-081923

6. Chen RE, Thorner J (2007) Function and regulation in MAPK signaling pathways: lessons learned from the yeast Saccharomyces cerevisiae. Biochim Biophys Acta 1773(8):1311-1340. https:// doi.org/10.1016/j.bbamcr.2007.05.003

7. Zhao X, Mehrabi R, Xu JR (2007) Mitogen-activated protein kinase pathways and fungal pathogenesis. Eukaryot Cell 6(10):1701-1714. https://doi.org/10.1128/EC.00216-07

8. Madhani HD, Styles CA, Fink GR (1997) MAP kinases with distinct inhibitory functions impart signaling specificity during yeast differentiation. Cell 91(5):673-684. https://doi.org/10.1016/S0092 -8674(00)80454-7

9. Chen J, Chen J, Lane S, Liu H (2002) A conserved mitogen-activated protein kinase pathway is required for mating in Candida 
albicans. Mol Microbiol 46(5):1335-1344. https://doi.org/10.10 46/j.1365-2958.2002.03249.x

10. Müller P, Aichinger C, Feldbrügge M, Kahmann R (1999) The MAP kinase kpp2 regulates mating and pathogenic development in Ustilago maydis. Mol Microbiol 34(5):1007-1017. https://doi. org/10.1046/j.1365-2958.1999.01661.x

11. Brachmann A, Schirawski J, Müller P, Kahmann R (2003) An unusual MAP kinase is required for efficient penetration of the plant surface by Ustilago maydis. EMBO J 22(9):2199-2210. https:// doi.org/10.1093/emboj/cdg198

12. Müller P, Weinzierl G, Brachmann A, Feldbrügge M, Kahmann R (2003) Mating and pathogenic development of the smut fungus Ustilago maydis are regulated by one mitogen-activated protein kinase cascade. Eukaryot Cell 2(6):1187-1199. https://doi. org/10.1128/EC.2.6.1187-1199.2003

13. Banuett F, Herskowitz I (1994) Identification of fuz7, a Ustilago maydis MEK/MAPKK homolog required for a-locus-dependent and-independent steps in the fungal life cycle. Genes Dev 8(12):1376-1378. https://doi.org/10.1101/gad.8.12.1367

14. Kaffarnik F, Müller P, Leibundgut M, Kahmann R, Feldbrügge M (2003) PKA and MAPK phosphorylation of Prf1 allows promoter discrimination in Ustilago maydis. EMBO J 22(21):5817-5826. https://doi.org/10.1093/emboj/cdg554

15. Hartmann HA, Kahmann R, Bölker M (1996) The pheromone response factor coordinates filamentous growth and pathogenicity in Ustilago maydis. EMBO J 15(7):1632-1641

16. Lanver D, Mendoza-Mendoza A, Brachmann A, Kahmann R (2010) Sho1 and Msb2-related proteins regulate appressorium development in the smut fungus Ustilago maydis. Plant Cell 22(6):2085-2101. https://doi.org/10.1105/tpc.109.073734

17. Di Stasio M, Brefort T, Mendoza-Mendoza A, Münch K, Kahmann R (2009) The dual specificity phosphatase Rok1 negatively regulates mating and pathogenicity in Ustilago maydis. Mol Microbiol 73(1):73-88. https://doi.org/10.1111/j.1365-2958.2009.06747.x

18. Kahmann R, Kämper J (2004) Ustilago maydis: how its biology relates to pathogenic development. New Phytol 164(1):31-42. https://doi.org/10.1111/j.1469-8137.2004.01156.x
19. Zhang Y, Cao Q, Hu P, Cui H, Yu X, Ye Z (2017) Investigation on the differentiation of two ustilago esculenta strains-implications of a relationship with the host phenotypes appearing in the fields. BMC Microbiol 17(1):228-243. https://doi.org/10.1186/ s12866-017-1138-8

20. Livak KJ, Schmittgen TD (2001) Analysis of relative gene expression data using real-time quantitative PCR and the $2^{-\triangle \Delta C T}$ method. Methods 25(4):402-408. https://doi.org/10.1006/meth.2001.1262

21. Arai R, Ueda H, Kitayama A, Kamiya N, Teruyuki N (2001) Design of the linker which effectively separate domains of a bifunctional fusion protein. Protein Eng 14(8):529-532. https:// doi.org/10.1093/protein/14.8.529

22. Yu J, Zhang Y, Cui H, Hu P, Yu X, Ye Z (2015) An efficient genetic manipulation protocol for Ustilago esculenta. FEMS Microbiol Lett 362(12):fnv087. https://doi.org/10.1093/femsle/ fnv087

23. Hunter T, Plowman GD (1997) The protein kinases of budding yeast: six score and more. Trends Biochem Sci 22(1):18-22. https ://doi.org/10.1016/S0968-0004(96)10068-2

24. Mayorga ME, Gold SE (1999) A MAP kinase encoded by the $u b c 3$ gene of Ustilago maydis is required for filamentous growth and full virulence. Mol Microbiol 34(3):485-497. https://doi.org/10. 1046/j.1365-2958.1999.01610.x

25. Xu JR, Hamer JE (1996) MAP kinase and cAMP signaling regulate infection structure formation and pathogenic growth in the rice blast fungus Magnaporthe grisea. Genes Dev 10(21):26962706. https://doi.org/10.1101/gad.10.21.2696

26. Elion EA, Grisafi PL, Fink GR (1990) FUS3 encodes a cdc2+/ CDC28-related kinase required for the transition from mitosis into conjugation. Cell 60(4):649-664. https://doi.org/10.1016/00928674(90)90668-5

27. Csank C, Schröppel K, Leberer E, Harcus D, Mohamed O, Meloche S, Thomas DY, Whiteway M (1998) Roles of the Candida albicans mitogen-activated protein kinase homolog, Cek1p, in hyphal development and systemic candidiasis. Infect Immun 66(6):2713-2721 